\title{
Real-world experience with dalbavancin therapy in gram-positive skin and soft tissue infection, bone and joint infection
}

\author{
Selma Tobudic ${ }^{1}\left[\right.$ Christina Forstner $^{1,2} \cdot$ Heinz Burgmann $^{1} \cdot$ Heimo Lagler ${ }^{1} \cdot$ Christoph Steininger $^{1} \cdot$ Ludwig Traby $^{1}$. \\ Mathias G. Vossen ${ }^{1} \cdot$ Stefan Winkler ${ }^{1} \cdot$ Florian Thalhammer $^{1}$
}

Received: 18 June 2019 / Accepted: 30 August 2019 / Published online: 13 September 2019

(C) The Author(s) 2019

\begin{abstract}
Purpose Dalbavancin is a novel lipoglycopeptide with potent activity against several gram-positive pathogens, an excellent safety profile and a long elimination half-life.

Methods In this case series observed at the University Hospital of Vienna between 2015 and 2017, all adult patients with gram-positive infections who received at least one dosage of dalbavancin were screened $(n=118)$. A total of 72 patients were included in the final analysis. The number of included patients stratified by the source of infection was: skin and soft tissue infection (SSTI) $(n=26)$, osteomyelitis $(n=20)$, spondylodiscitis $(n=14)$, acute septic arthritis $(n=4)$ and prosthetic joint infection $(n=8)$.

Results In 46 patients (64\%), clinical cure was detected at the end of dalbavancin therapy without additional antibiotic therapy. Of the 26 patients who received additional antibiotic therapy other than dalbavancin, 15 patients (21\%) showed no clinical improvement under dalbavancin therapy, four patients (5\%) had side effects (nausea $n=1$, exanthema $n=2$, hyperglycemia $n=1$ ), and in seven patients (10\%) clinical improvement under dalbavancin therapy was detected but antibiotic therapy was de-escalated to an oral drug.

Conclusion We demonstrated high clinical effectiveness of dalbavancin for acute gram-positive infections primarily acute SSTI, acute septic arthritis, acute osteomyelitis and spondylodiscitis. In patients with biofilm-associated infection (chronic infection or joint prosthesis), source control was absolutely necessary for treatment success.
\end{abstract}

Keywords Dalbavancin · SSTI · Osteomyelitis · Vertebral osteomyelitis · Septic arthritis · Prosthetic joint infections

\section{Introduction}

Gram-positive bacteria are among the most important human pathogens associated with community-acquired and health-care-associated infections [1]. The most common gram-positive infections, which remain a substantial challenge, include skin and soft tissue infections (SSTIs) and wound infections, bloodstream infections, pneumonia, osteomyelitis, vertebral osteomyelitis, infective endocarditis, and device-related infections [2]. Dalbavancin is a

Selma Tobudic

selma.tobudic@meduniwien.ac.at

1 Division of Infectious Diseases and Tropical Medicine, Department of Medicine I, Medical University of Vienna, Waehringer Guertel 18-20, 1090 Vienna, Austria

2 Institute Infectious Diseases and Infection Control, Jena University Hospital, Am Klinikum 1, 07747 Jena, Germany novel lipoglycopeptide approved by European Medicines Agency (EMA) and the US Food and Drug Administration for the treatment of acute bacterial skin and skin structure infections (ABSSSIs) in adults [3]. It is approved for the treatment of dalbavancin-susceptible gram-positive bacteria, including methicillin-resistant Staphylococcus aureus and strains with intermediate sensitivity to vancomycin [4]. In vitro studies showed good activity of dalbavancin against several gram-positive pathogens, including Staphylococcus aureus (S. aureus), Streptococcus agalactiae, Streptococcus pyogenes, Streptococcus anginosus, Enterococcus faecium, and Enterococcus faecalis, although clinical data regarding the clinical outcome in enterococcal infections are limited [5-8]. Resistance to staphylococci is rare, being reported in less than $1 \%$ of isolates $[6,9]$, but dalbavancin demonstrated poor activity against vancomycin-resistant $S$. aureus (VRSA) and no activity against VanA phenotype-resistant enterococci $[10,11]$. Dalbavancin exhibits linear, dose-dependent 
pharmacokinetics with an elimination half-life of approximately $346 \mathrm{~h}$, allowing a prolonged interval between two doses [12-15]. The approved dosage for dalbavancin for adults with ABSSSIs is a two-dose regimen of $1000 \mathrm{mg}$ on day 1 followed by $500 \mathrm{mg}$ on day 8 and single use of $1500 \mathrm{mg}[3,8,16]$. Due additionally, dalbavancin doseregimens used in our university hospital include a two-dose regimen of $1500 \mathrm{mg}$ on day 1 followed by $1000 \mathrm{mg}$ on day 14 [17, 18], and a two dose-regimen of $1500 \mathrm{mg}$ on day 1 followed by $1500 \mathrm{mg}$ on day $8[7,16,19,20]$. The prolonged use of dalbavancin for several weeks is well established in our clinical setting [7].

In the present study, clinical outcomes and safety of dalbavancin in the treatment of gram-positive infections in adults, such as skin and soft tissue infection (SSTI), osteomyelitis, vertebral osteomyelitis, prosthetic joint infection and acute septic arthritis, were retrospectively evaluated.

\section{Materials and methods}

This case series was performed at the University Hospital of Vienna, Austria, from January 2015 to December 2017. After approval by the local ethics committee in 2017 (No. 1445/2017), we retrospectively screened all adult patients treated by at least one dose of dalbavancin for SSTI, osteomyelitis, vertebral osteomyelitis, acute septic arthritis and prosthetic joint infection. SSTI was defined as acute or chronic. Acute SSTI was defined as erysipelas, major abscess, traumatic wound or surgical site infection. Erysipelas was defined by physicians using clinical and microbiological criteria. The need of a patient informed consent was waived by the local ethics committee due to the retrospective nature of the present study.

Patient demographics, causative pathogens, antimicrobial therapy employed in the treatment, and overall clinical outcome as judged by the investigators were collected. Dalbavancin data included indication, treatment regimen (regimen 1: $1000 \mathrm{mg}$ on first day and $500 \mathrm{mg}$ every 7 days, regimen 2 : $1500 \mathrm{mg}$ on first day and $1000 \mathrm{mg}$ every 14 days, regimen 3 : $1500 \mathrm{mg}$ on first day, and on day 8 and in individual cases repetition of $1500 \mathrm{mg}$ on days 56 and 63), duration of therapy and reporting of side effects [7]. The duration of therapy for a single regimen was calculated using the number of administrated dalbavancin doses: time (weeks) for regimen $1=$ number of administrated doses $\times 1$; time (weeks) for regimen $2=$ number of administrated doses $\times 2$; time (weeks). For regimen 3, the use of $1500 \mathrm{mg}$ dalbavancin on day 1 , followed by dalbavancin $1500 \mathrm{mg}$ on day 8 was calculated as 8 -week therapy. In case of prolonged therapy and use of $1500 \mathrm{mg}$ on day 53 and day 64, duration of dalbavancin therapy was calculated as 16 weeks. The primary endpoint was defined as clinical cure or failure. Clinical cure was defined as resolution of all clinical signs and symptoms of infection, no additional antibiotic therapy required for the indication initially treated with dalbavancin and no microbiological relapse during the follow-up period of 6 months after completion of treatment. Failure of dalbavancin therapy was defined as no clinical improvement or worsening of current infection or new/recurrent signs and symptoms of infection requiring either a change or addition of antibiotic therapy, or microbiological relapse within 6 months after completion of (initial) treatment [7]. Change of antibiotic therapy was stratified by cause: no clinical improvement, side effect and antibiotic de-escalation to an oral drug. As secondary endpoint, number of patients (\%) with clinical failure under dalbavancin therapy but resolution of clinical symptoms after antibiotic change in follow-up of 6 months was determined. Further secondary endpoints were defined as duration of dalbavancin therapy in weeks, number (\%) of patients treated with combination therapy, number (\%) of patients treated with dalbavancin as sequential therapy and side effects. Creatinine ( $\mathrm{mg} / \mathrm{dl})$ as a measure of kidney function and liver enzymes (GPT and GOT, U/l) as a measure of hepatotoxicity were monitored at least at the beginning and the end of the dalbavancin therapy (7-14 days after last dose). Dalbavancin outcome was stratified by the causative pathogen isolated at the site of infection.

\section{Results}

\section{Demographic data}

In the final analysis, we included 38 men and 34 women with a median age of 56.5 years (range 18-92 years). The number of included patients stratified by the source of infection was: SSTI $(n=26)$, osteomyelitis $(n=20)$, vertebral osteomyelitis $(n=14)$, acute septic arthritis $(n=4)$ and prosthetic joint infection $(n=8)$. Table 1 shows the types of SSTI, cause of osteomyelitis and vertebral osteomyelitis, surgical interventions in the case of osteomyelitis, and the treatment strategies in the case of prosthetic joint infections.

\section{Follow-up and clinical outcome}

A total of 118 patients with gram-positive infections and at least one dosage of dalbavancin were screened and in 46 patients, long-term follow-up was not available due to an incomplete medical record (missing outcome data $n=23$, missing microbiological data $n=9$, missing documentation of exposure $n=14$ ). These patients were excluded from further analyses. In the final analysis, 72 patients were included. In 46 patients (64\%), clinical cure was detected under dalbavancin therapy without subsequent antibiotic therapy for the initial gram-positive infection. Of the 26 patients who were 
Table 1 Patient and disease characteristics $(n=72)$

\begin{tabular}{|c|c|}
\hline Age, years, median (range) & $56.5(18-92)$ \\
\hline Male sex $n(\%)$ & $38(53)$ \\
\hline SSTI, $n(\%)$ & $26(36)$ \\
\hline Acute & 21 \\
\hline Chronic & 5 \\
\hline Osteomyelitis, $n(\%)$ & $20(28)$ \\
\hline \multicolumn{2}{|l|}{ Cause of osteomyelitis } \\
\hline Postoperative & 8 \\
\hline Diabetic foot & 9 \\
\hline Skin ulcera & 1 \\
\hline Dental implant & 1 \\
\hline Unknown & 1 \\
\hline \multicolumn{2}{|l|}{ Surgical intervention } \\
\hline Amputation & 3 \\
\hline Debridement & 2 \\
\hline Partial resection & 1 \\
\hline Vertebral osteomyelitis, $n(\%)$ & $14(19)$ \\
\hline \multicolumn{2}{|l|}{ Cause of vertebral osteomyelitis } \\
\hline Postoperative & 1 \\
\hline Hematogenous spread & 8 \\
\hline Spine fracture by accident & 1 \\
\hline Unknown & 4 \\
\hline Acute septic arthritis, $n(\%)$ & $4(6)$ \\
\hline Prosthetic joint infections, $n(\%)$ & $8(11)$ \\
\hline \multicolumn{2}{|l|}{ Treatment strategy } \\
\hline Total prosthesis replacement & 4 \\
\hline Amputation & 1 \\
\hline Debridement and change of mobile parts & 1 \\
\hline No source control & 2 \\
\hline
\end{tabular}

$n$ number, SSTI skin and soft tissue infection

switched to other antimicrobial treatment, 15 patients (21\%) showed no clinical improvement under dalbavancin therapy, 4 patients (5\%) had side effects (nausea $n=1$, exanthema $n=2$, hyperglycemia $n=1$ ), and in seven patients $(10 \%)$, clinical improvement under dalbavancin was detected but therapy was changed to an alternative treatment other than dalbavancin due to the possibility to use an oral drug. Twelve of the 15 patients without clinical improvement under dalbavancin therapy (80\%), also failed to achieve any clinical improvement after change to an alternative antibiotic regimen (Fig. 1). Dalbavancin was used in 14 patients (19\%) as primary regime, in 39 patients (54\%) as salvage treatment and in 19 patients (27\%) as simplification due to outpatient parenteral treatment option.

\section{Clinical outcomes stratified by source of infection}

In patients with SSTI, clinical cure with dalbavancin therapy was detected in 20 of 26 patients (77\%). In six patients, therapy was switched to a different antibiotic treatment (no clinical improvement $n=5$, side effects $n=1$ ). Resolution of clinical symptoms after antibiotic change was observed in two patients.

Of 20 patients with osteomyelitis, 13 (65\%) patients had clinical resolution of their osteomyelitis with dalbavancin therapy. Only in one patient, resolution of clinical symptoms was achieved after change of antibiotic regimen. In 6 of 13 patients with osteomyelitis and clinical cure (46\%), antibiotic therapy was combined with surgical intervention (debridement or amputation).

Seven of 14 patients (50\%) with vertebral osteomyelitis had no clinical signs of infection under dalbavancin therapy and did not require further antibiotic treatment in the follow-up period of 6 months. The reasons for therapy change were no clinical improvement under dalbavancin therapy $(n=1)$, antibiotic de-escalation to an oral drug $(n=4)$ and side effects $(n=2)$. After antibiotic change to another regimen, resolution of clinical symptoms and signs of infection was achieved in all seven patients. All patients with acute septic arthritis $(n=4)$ showed clinical cure with dalbavancin therapy.

In patients with prosthetic joint infections, clinical cure as final outcome was documented in six of eight patients (75\%) treated by combination of antibiotic therapy and surgical intervention (three-stage total prosthesis replacement (TPR) $n=1$, two-stage TPR $n=2$, one-stage TPR $n=1$, amputation $n=1$, debridement and change of mobile parts $n=1$ ). In three of six patients, dalbavancin therapy was deescalated to an oral drug. In two patients without surgical treatment and with previous long-term antibiotic therapy, dalbavancin achieved no clinical improvement (Table 2).

Table 3 includes all patients with no clinical improvement under dalbavancin therapy. Thirteen of 15 patients (87\%) showed no clinical improvement with alternative antibiotics due to missing or incomplete source control (chronic wound, osteomyelitis, prosthetic joint infection). Diabetes mellitus was the most common comorbidity in this patient group.

Median duration (range) of dalbavancin therapy was: 2 weeks (2-16) for SSTI, 8 weeks (4-32) for osteomyelitis, 9 weeks (2-16) for vertebral osteomyelitis, 3.5 weeks (2-10) for acute septic arthritis and 12 weeks (6-32) for prosthetic joint infections. The most common regimen used was regimen 2 (initial dose $1500 \mathrm{mg}$ and $1000 \mathrm{mg}$ each 14 days) in $71 \%$ of patients. Clinical cure was seen in 11 of 17 patients $(65 \%)$ using regimen 1 , in 31 of 51 patients $(61 \%)$ using regimen 2 and in 4 of 5 patients (80\%) using regimen 3 . All four patients with side effects were treated with regimen 2. In patients with osteomyelitis, prosthetic joint infection, vertebral osteomyelitis and acute septic arthritis dalbavancin was mainly used as sequential therapy $(100 \%, 67 \%, 86 \%$, and $100 \%$, respectively). Dalbavancin was used as monotherapy in the majority of patients $(n=57,79 \%)$ (Table 4$)$. 


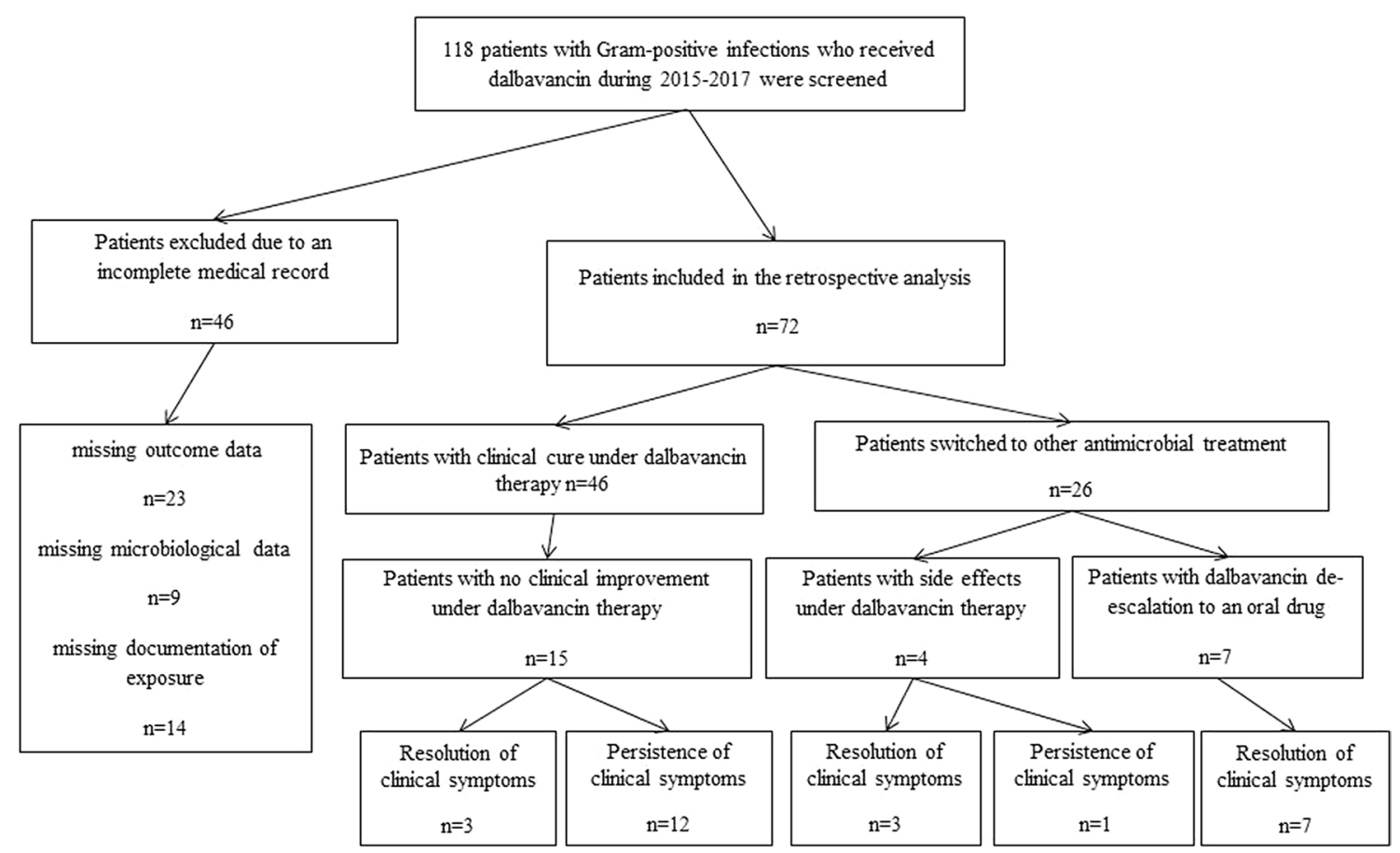

Fig. 1 Flow chart of the study population including follow up and clinical outcome

Table 2 Outcome of all patients after 6-month follow-up $(n=72)$ treated with dalbavancin

\begin{tabular}{|c|c|c|c|}
\hline Indication & $\begin{array}{l}\text { Number of patients }(\%) \\
\text { with clinical cure under } \\
\text { DALB }\end{array}$ & $\begin{array}{l}\text { Number of patients }(\%) \text { with antibi- } \\
\text { otic change stratified by reasons for } \\
\text { change }\end{array}$ & $\begin{array}{l}\text { Number of patients }(\%) \text { with clinical } \\
\text { failure under DALB therapy but } \\
\text { resolution of clinical symptoms after } \\
\text { antibiotic change }\end{array}$ \\
\hline $\operatorname{SSTI}(n=26)$ & $20(77)$ & $\begin{array}{l}\text { No clinical improvement, } n=5 \text { (19) } \\
\text { Side effects, } n=1 \text { (4) }\end{array}$ & $2(8)$ \\
\hline Acute $(n=21)$ & $20(95)$ & No clinical improvement, $n=1$ (5) & $1(5)$ \\
\hline Chronic $(n=5)$ & $0(0)$ & $\begin{array}{l}\text { No clinical improvement, } n=4 \text { (80) } \\
\text { Side effects, } n=1(20)\end{array}$ & $1(20)$ \\
\hline Osteomyelitis, all $(n=20)$ & $12(60)$ & $\begin{array}{l}\text { No clinical improvement, } n=7 \text { (35) } \\
\text { Side effects, } n=1 \text { (5) }\end{array}$ & $1(5)$ \\
\hline $\begin{array}{l}\text { Acute postoperative osteomyelitis } \\
\quad(n=7)\end{array}$ & $7(100)$ & - & 0 \\
\hline Chronic osteomyelitis $(n=13)$ & $5(38)$ & $\begin{array}{l}\text { No clinical improvement, } n=7 \text { (54) } \\
\text { Side effects, } n=1 \text { (8) }\end{array}$ & $1(8)$ \\
\hline Vertebral osteomyelitis $(n=14)$ & $7(50)$ & $\begin{array}{l}\text { No clinical improvement, } n=1(7) \\
\text { Antibiotic de-escalation to an oral } \\
\text { drug, } n=4(29) \\
\text { Side effects } n=2(14)\end{array}$ & $7(50)$ \\
\hline Acute septic arthritis $(n=4)$ & $4(100)$ & - & 0 \\
\hline Prosthetic joint infection $(n=8)$ & $3(38)$ & $\begin{array}{l}\text { No clinical improvement, } n=2(25) \\
\text { Antibiotic de-escalation to an oral } \\
\text { drug, } n=3(38)\end{array}$ & $3(38)$ \\
\hline
\end{tabular}

SSTI skin and soft tissue infection, $D A L B$ dalbavancin

No significant increase in creatinine $(>1.5 \times$ increase) and liver enzyme ( $>3 \times$ increase) values between start and end of therapy was found.

\section{Clinical outcome stratified by pathogen}

Dalbavancin was most commonly applied in patients with staphylococcal infection (MSSA 25\%, MRSA 8\%, MSSE 
Table 3 Characteristic of patients with clinical failure of dalbavancin treatment $(n=15)$

\begin{tabular}{|c|c|c|c|c|c|c|}
\hline Indication & Infected site & Pathogen & $\begin{array}{l}\text { Prior other } \\
\text { antibiotic } \\
\text { therapy }\end{array}$ & $\begin{array}{l}\text { Concomitant anti- } \\
\text { biotic }\end{array}$ & $\begin{array}{l}\text { Resolution of clini- } \\
\text { cal symptoms after } \\
\text { switch from DALB } \\
\text { to other treatment }\end{array}$ & Comorbidity \\
\hline CWI & Foot & MRSA & Yes & No & No & IDDM PAOD \\
\hline CWI & Hip & MSSE & Yes & No & No & NIDDM \\
\hline CWI & Abdomen & MRSA & Yes & Moxifloxacin & No & Acne inversa \\
\hline CWI & Inguinal & MSSA & Yes & No & No & Acne inversa \\
\hline $\begin{array}{l}\text { Postoperative acute } \\
\text { SSTI }\end{array}$ & Breast & MRSA & Yes & No & Yes & Breast cancer \\
\hline $\mathrm{COM}$ & Maxilla & E. faecalis & Yes & No & No & Dental implant \\
\hline $\mathrm{COM}$ & Metatarsal & MRSE & Yes & Ciprofloxacin & No & NIDDM PAOD \\
\hline $\mathrm{COM}$ & Metatarsal & $\begin{array}{l}\text { E. faecalis, S. san- } \\
\text { guinis }\end{array}$ & Yes & No & No & NIDDM PAOD \\
\hline $\mathrm{COM}$ & Metatarsal & $\begin{array}{l}\text { MSSA, Streptococ- } \\
\text { cus spp. }\end{array}$ & Yes & No & No & IDDM \\
\hline $\mathrm{COM}$ & Metatarsal & MRSA & Yes & No & No & NIDDM Lymphoma \\
\hline $\mathrm{COM}$ & Tibia & MSSA & Yes & Moxifloxacin & No & $\begin{array}{l}\text { PAOD cigarette } \\
\text { smoking }\end{array}$ \\
\hline $\mathrm{COM}$ & Tibia & MSSE & Yes & No & Yes & NIDDM; PAOD \\
\hline $\mathrm{COM}$ & Calcaneus & MSSA & Yes & Clindamycin & No & IDDM \\
\hline $\begin{array}{l}\text { Vertebral osteomy- } \\
\text { elitis }\end{array}$ & Lumbal & C. acnes & Yes & No & Yes & No \\
\hline $\begin{array}{l}\text { Prosthetic joint infec- } \\
\text { tions }\end{array}$ & Hip & No pathogen found & Yes & Levofloxacin & No & No \\
\hline $\begin{array}{l}\text { Prosthetic joint infec- } \\
\text { tions }\end{array}$ & Knee & S. anginosus & Yes & No & No & No \\
\hline
\end{tabular}

C. acnes Cutibacterium acnes, COM chronic osteomyelitis, CWI chronic wound infection, DALB dalbavancin, E. faecalis Enterococcus faecalis, IDDM insulin-dependent diabetes mellitus, MRSA Methicillin-resistant Staphylococcus aureus, MSSA Methicillin-sensitive Staphylococcus aureus, MSSE Methicillin-sensitive Staphylococcus epidermidis, NIDDM non-insulin-dependent diabetes mellitus, PAOD peripheral arterial occlusive disease, S. sanguinis Streptococcus sanguinis, SSTI skin and soft tissue infection

$7 \%$ and MRSE 4\%), followed by streptococcal infection and infection caused by Enterococcus faecalis (E. faecalis).

Table 5 shows the outcome of patients stratified by the causative pathogen.

\section{Discussion}

This case series provides real-life experience of dalbavancin use for acute and chronic SSTI and for other offlabel indications including bone and joint infections in our hospital. The main results of our study were that we found good safety of dalbavancin for short- and long-term treatment and high clinical cure rates in acute infection including erysipelas, acute septic arthritis, acute vertebral osteomyelitis, and acute osteomyelitis, but limited treatment success among chronic infections, particularly in cases with insufficient source control. In the last 3 years, several studies reported experiences with dalbavancin use $[7,8,19,21]$. In the present work, the overall clinical cure rate $(64 \%)$ under dalbavancin was lower compared with previous reports ( $84.1 \%$ by Bouza et al. and $89.5 \%$ by Wunsch et al.) $[8,19]$. Noteworthy, in our case series, dalbavancin was used mainly as salvage treatment (54\%) due to missing clinical success of the prior antimicrobial regimen and in off-label indications (54 patients, 75\%). Only 14 patients (19\%) received dalbavancin as primary treatment regimen. Additionally, it is known that change of antibiotic regimen occurs in clinical practice due to different reasons apart from missing clinical response. In fact, no clinical improvement was detected in 15 patients under dalbavancin, and only in 3 of those 15 patients (21\%), clinical cure could be achieved by another antimicrobial regimen.

The management of bone and joint infection or of patients with chronic SSTI is very complex due to the biofilm life of bacteria and clinical success can only be achieved by source control in most cases [22]. Recently, high in vitro activity of dalbavancin against MRSA, MRSE and enterococcal biofilms was reported [23, 24]. However, in the present study, treatment success was particularly low in MRSA infections. 
Table 4 Therapy regimen, duration of therapy in weeks, number of patients treated with combination therapy (dalbavancin + other antibiotic) and number of patients treated with dalbavancin as sequential therapy

\begin{tabular}{|c|c|c|c|c|}
\hline Indication & $\begin{array}{l}\text { Therapy } \\
\text { regimen }^{\mathrm{a}}(\mathrm{n})\end{array}$ & $\begin{array}{l}\text { Duration of DALB } \\
\text { therapy (weeks) } \\
\text { Median (range) }\end{array}$ & $\begin{array}{l}\text { Number }(\%) \text { of patients treated with } \\
\text { combination therapy (DALB + other AB) }\end{array}$ & $\begin{array}{l}\text { Number (\%) of patients treated } \\
\text { with DALB as sequential therapy }\end{array}$ \\
\hline $\begin{array}{l}\text { SSTI } \\
n=26\end{array}$ & $\begin{array}{l}1(9) \\
2(16) \\
3(1)\end{array}$ & $2(2-10)$ & $2(8)$ & $11(42)$ \\
\hline $\begin{array}{l}\text { Osteomyelitis } \\
n=20\end{array}$ & $\begin{array}{l}1(2) \\
2(17) \\
3(1)\end{array}$ & $8(4-32)$ & $5(25)$ & $20(100)$ \\
\hline $\begin{array}{l}\text { Vertebral osteomyelitis } \\
n=14\end{array}$ & $\begin{array}{l}1(3) \\
2(9) \\
3(3)\end{array}$ & $9(2-16)$ & $5(36)$ & $12(86)$ \\
\hline $\begin{array}{l}\text { Acute septic arthritis } \\
n=4\end{array}$ & $\begin{array}{l}1(2) \\
2(2) \\
3(0)\end{array}$ & $3.5(2-10)$ & $1(25)$ & $4(100)$ \\
\hline $\begin{array}{l}\text { Prosthetic joint infections } \\
n=8\end{array}$ & $\begin{array}{l}1(1) \\
2(7) \\
3(0)\end{array}$ & $12(6-32)$ & $2(33)$ & $4(67)$ \\
\hline
\end{tabular}

$A B$ antibiotic, $D A L B$ dalbavancin, $n$ number, SSTI skin and soft tissue infection

${ }^{a}$ Therapy regimen 1: $1000 \mathrm{mg}$ on first day and $500 \mathrm{mg}$ every 7 days; therapy regimen $2: 1500 \mathrm{mg}$ on first day and $1000 \mathrm{mg}$ every 14 days; therapy regimen 3: $1500 \mathrm{mg}$ on first day, and on day 8 and in individual cases repetition of $1500 \mathrm{mg}$ on days 56 and on day 63

Furthermore, treatment success was low in patients with diabetic foot syndrome (40\%), with prolonged use of dalbavancin alone or in combination not being superior compared with other antibiotics. In patients with chronic osteomyelitis, clinical cure rates were $39 \%$ under dalbavancin and $46 \%$ after changing to a further regimen. In a recently published randomized clinical trial, dalbavancin achieved response rate as high as $97 \%$ in patients with osteomyelitis [16]. However, in this trial, dalbavancin was used as primary option for patients with a first episode of osteomyelitis and not for chronic cases. In accordance, we also detected a high clinical cure of $100 \%$ in patients with acute osteomyelitis.

For vertebral osteomyelitis, a randomized controlled trial demonstrated non-inferiority of 6-week compared with 12 -week antibiotic therapy and very recently, it was shown that an early change to an oral drug is non-inferior to intravenous therapy in stable patients $[25,26]$. In the present study, dalbavancin was a good treatment option when used as primary or sequential therapy in patients with microbiologically, radiologically, or histologically documented vertebral osteomyelitis. Only in a single patient, treatment was switched from dalbavancin to another antibiotic therapy due to missing clinical response after 6 weeks.

All patients with acute septic arthritis treated with dalbavancin showed clinical cure, but it has to be noted that all patients received dalbavancin as sequential therapy. The most important therapy for prosthetic joint infection is adequate source control including surgical and antibiotic therapy. Similarly as in other studies, for prosthetic joint infections, clinical success under dalbavancin was only reached when used in combination with surgical treatment [27].

The incidence of adverse events observed in this study was similar to that reported in randomized, controlled clinical trials and in real-life reports [7, 8, 16, 19, 21]. In our experience, dalbavancin had an excellent safety profile with no significant drug interactions. Patients were followed for 6 months after the last dose of dalbavancin. We observed acute reactions such as exanthema or hyperglycemia, but detected no long-term negative effects.

In terms of duration of dalbavancin therapy, patients in our hospital received prolonged therapy using different regimens. The rationale of regimen 2 over regimen 1 is to minimize the frequency of intravenous therapy, while maximizing the initial exposure. Initial dose (loading dose) $1500 \mathrm{mg}$ is an approved dose and due to the long half-life $>14$ days, a single maintenance dose of $1000 \mathrm{mg}$ every 2 weeks is sufficient to exceed the MIC90 for target gram-positive pathogens. However, if a treatment duration $\geq 6$ weeks is planned, then regime 3 is the better option [17, 18]. Our case series has some limitations as other similar studies based on reallife experience due to the retrospective analysis. First of all, the study conclusions are limited due to small number of patients. Second, long-term follow-up was not available in all cases. The decision to start with dalbavancin therapy or to switch to other drugs was mainly taken by physicians; therefore, there were no uniform criteria for using the drug.

In conclusion, we report excellent safety and high clinical effectiveness of dalbavancin for acute gram-positive 
Table 5 Outcome of dalbavancin therapy stratified by species

\begin{tabular}{ll}
\hline Pathogen & All $(n=72)$ \\
\hline MSSA $n(\%)$ & $27(38)$ \\
Cure (DALB outcome) $n(\%)$ & $18(67)$ \\
Resolution of clinical symptoms after switch from DALB to other treatment $n(\%)$ & $5(19)$ \\
MRSA $n(\%)$ & $6(8)$ \\
Cure (DALB outcome) $n(\%)$ & $0(0)$ \\
Resolution of clinical symptoms after switch from DALB to other treatment $n(\%)$ & $1(17)$ \\
MSSE $n(\%)$ & $5(7)$ \\
Cure (DALB outcome) $n(\%)$ & $3(60)$ \\
Resolution of clinical symptoms after switch from DALB to other treatment $n(\%)$ & $1(20)$ \\
MRSE $n(\%)$ & $3(4)$ \\
Cure (DALB outcome) $n(\%)$ & $3(100)$ \\
Resolution of clinical symptoms after switch from DALB to other treatment $n(\%)$ & 0 \\
Enterococcus spp. $n(\%)$ & $3(4)$ \\
Cure (DALB outcome) $n(\%)$ & $2(67)$ \\
Resolution of clinical symptoms after switch from DALB to other treatment $n(\%)$ & 0 \\
Streptococcus spp. $n(\%)$ & $11(15)$ \\
Cure (DALB outcome) $n(\%)$ & $6(55)$ \\
Resolution of clinical symptoms after switch from DALB to other treatment $n(\%)$ & $4(36)$ \\
Cutibacterium acnes $n(\%)$ & $3(4)$ \\
Cure (DALB outcome) $n(\%)$ & $2(67)$ \\
Resolution of clinical symptoms after switch from DALB to other treatment $n(\%)$ & $1(33)$ \\
Mixed infection $n(\%)$ & $4(6)$ \\
Cure (DALB outcome) $n(\%)$ & $3(75)$ \\
Resolution of clinical symptoms after switch from DALB to other treatment $n(\%)$ & 0 \\
Unknown $n(\%)$ & $10(14)$ \\
Cure (DALB outcome) $n(\%)$ & $9(90)$ \\
Resolution of clinical symptoms after switch from DALB to other treatment $n(\%)$ & 0 \\
\hline & \\
\hline
\end{tabular}

DALB dalbavancin, MRSA Methicillin-resistant Staphylococcus aureus, MRSE Methicillin-resistant Staphylococcus epidermidis, MSSA Methicillin-sensitive Staphylococcus aureus, MSSE Methicillin-sensitive Staphylococcus epidermidis, $n$ number infections primarily acute SSTI, acute septic arthritis, acute osteomyelitis and vertebral osteomyelitis. In patients with biofilm-associated infection (chronic infection or joint prosthesis), source control was absolutely necessary for treatment success.

Acknowledgements Open access funding provided by Medical University of Vienna.

\section{Compliance with ethical standards}

Conflict of interest The authors declare that they have no competing interest.

Open Access This article is distributed under the terms of the Creative Commons Attribution 4.0 International License (http://creativeco mmons.org/licenses/by/4.0/), which permits unrestricted use, distribution, and reproduction in any medium, provided you give appropriate credit to the original author(s) and the source, provide a link to the Creative Commons license, and indicate if changes were made.

\section{References}

1. Menichetti F. Current and emerging serious gram-positive infections. Clin Microbiol Infect. 2005;11:22-8.

2. Woodford N, Livermore DM. Infections caused by gram-positive bacteria: a review of the global challenge. J Infect. 2009;59:S4-16.

3. Dunne MW, Puttagunta S, Giordano P, Krievins D, Zelasky M, Baldassarre J. A randomized clinical trial of single-dose versus weekly dalbavancin for treatment of acute bacterial skin and skin structure infection. Clin Infect Dis. 2016;62:545-51.

4. David MZ, Dryden M, Gottlieb T, Tattevin P, Gould IM. Recently approved antibacterials for methicillin-resistant Staphylococcus aureus (MRSA) and other gram-positive pathogens: the shock of the new. Int J Antimicrob Agents. 2017;50:303-7.

5. Jones RN, Farrell DJ, Flamm RK, Sader HS, Dunne MW, Mendes RE. Surrogate analysis of vancomycin to predict susceptible categorization of dalbavancin. Diagn Microbiol Infect Dis. 2015;82:73-7.

6. Jones RN, Flamm RK, Sader HS. Surveillance of dalbavancin potency and spectrum in the United States (2012). Diagn Microbiol Infect Dis. 2013;76:122-3.

7. Tobudic S, Forstner C, Burgmann H, Lagler H, Ramharter $\mathrm{M}$, Steininger $\mathrm{C}$, et al. Dalbavancin as primary and 
sequential treatment for gram-positive infective endocarditis: 2 -year experience at the general hospital of vienna. Clin Infect Dis. 2018;67:795-8.

8. Bouza E, Valerio M, Soriano A, Morata L, Carus EG, RodriguezGonzalez C, et al. Dalbavancin in the treatment of different grampositive infections: a real-life experience. Int J Antimicrob Agents. 2018;51:571-7.

9. Jones RN, Sader HS, Flamm RK. Update of dalbavancin spectrum and potency in the USA: report from the SENTRY Antimicrobial Surveillance Program (2011). Diagn Microbiol Infect Dis. 2013;75:304-7.

10. Mendes RE, Castanheira M, Farrell DJ, Flamm RK, Sader HS Jones RN. Update on dalbavancin activity tested against grampositive clinical isolates responsible for documented skin and skin-structure infections in US and European hospitals (2011-13). J Antimicrob Chemother. 2016;71:276-8.

11. Biedenbach DJ, Bell JM, Sader HS, Turnidge JD, Jones RN. Activities of dalbavancin against a worldwide collection of 81,673 gram-positive bacterial isolates. Antimicrob Agents Chemother. 2009;53:1260-3

12. Leighton A, Gottlieb AB, Dorr MB, Jabes D, Mosconi G, VanSaders $\mathrm{C}$, et al. Tolerability, pharmacokinetics, and serum bactericidal activity of intravenous dalbavancin in healthy volunteers. Antimicrob Agents Chemother. 2004;48:940-5.

13. Dorr MB, Jabes D, Cavaleri M, Dowell J, Mosconi G, Malabarba A, et al. Human pharmacokinetics and rationale for once-weekly dosing of dalbavancin, a semi-synthetic glycopeptide. J Antimicrob Chemother. 2005;55:ii25-30.

14. Marbury T, Dowell JA, Seltzer E, Buckwalter M. Pharmacokinetics of dalbavancin in patients with renal or hepatic impairment. J Clin Pharmacol. 2009;49:465-76.

15. Dowell JA, Goldstein BP, Buckwalter M, Stogniew M, Damle B. Pharmacokinetic-pharmacodynamic modeling of dalbavancin, a novel glycopeptide antibiotic. J Clin Pharmacol. 2008;48:1063-8.

16. Rappo U, Puttagunta S, Shevchenko V, Shevchenko A, Jandourek A, Gonzalez PL, et al. Dalbavancin for the treatment of osteomyelitis in adult patients: a randomized clinical trial of efficacy and safety. Open Forum Infect Dis. 2019;6:ofy331.
17. Dunne MW, Puttagunta S, Sprenger CR, Rubino C, Van Wart S, Baldassarre J. Extended-duration dosing and distribution of dalbavancin into bone and articular tissue. Antimicrob Agents Chemother. 2015;59:1849-55.

18. Boucher HW, Wilcox M, Talbot GH, Puttagunta S, Das AF, Dunne MW. Once-weekly dalbavancin versus daily conventional therapy for skin infection. N Engl J Med. 2014;370:2169-79.

19. Wunsch S, Krause R, Valentin T, Prattes J, Janata O, Lenger A, et al. Multicenter clinical experience of real life dalbavancin use in gram-positive infections. Int J Infect Dis. 2019;81:210-4.

20. Jandourek Alena SV, Schvenko O, Rappo U, Gonzalez P, Puttagunta S, Dunne M, Miceli R, Kovacevic M, De Bock G. Dalbavancin for the treatment of osteomyelitis in adult patients. Eur Congr Clin Microbiol Infect Dis. 2017.

21. Morata L, Cobo J, Fernandez-Sampedro M, Vasco PG, Ruano E, Lora-Tamayo J, et al. Safety and efficacy of prolonged use of dalbavancin in bone and joint infections. Antimicrob Agents Chemother. 2019;63(5):e02280-18. https://doi.org/10.1128/AAC.02280 -18 .

22. Del Pozo JL. Biofilm-related disease. Expert Rev Anti Infect Ther. 2018;16:51-65.

23. Knafl D, Tobudic S, Cheng SC, Bellamy DR, Thalhammer F. Dalbavancin reduces biofilms of methicillin-resistant Staphylococcus aureus (MRSA) and methicillin-resistant Staphylococcus epidermidis (MRSE). Eur J Clin Microbiol Infect Dis. 2017;36:677-80.

24. Neudorfer K, Schmidt-Malan SM, Patel R. Dalbavancin is active in vitro against biofilms formed by dalbavancin-susceptible enterococci. Diagn Microbiol Infect Dis. 2018;90:58-63.

25. Bernard L, Dinh A, Ghout I, Simo D, Zeller V, Issartel B, et al. Antibiotic treatment for 6 weeks versus 12 weeks in patients with pyogenic vertebral osteomyelitis: an open-label, non-inferiority, randomised, controlled trial. Lancet. 2015;385:875-82.

26. Li HK, Rombach I, Zambellas R, Walker AS, McNally MA, Atkins BL, et al. Oral versus intravenous antibiotics for bone and joint infection. N Engl J Med. 2019;380:425-36.

27. Zimmerli W, Trampuz A, Ochsner PE. Prosthetic-joint infections. N Engl J Med. 2004;351:1645-54. 